\title{
EFEITO DE DIFERENTES SUBSTRATOS SOBRE O DESENVOLVIMENTO DE MUDAS DE Acacia sp. ${ }^{1}$
}

\author{
Alexson de Mello Cunha², Gláucio de Mello Cunha ${ }^{3}$, Renato de Almeida Sarmento ${ }^{4}$, Glaucia de Mello \\ Cunha ${ }^{5}$ e José Francisco Teixeira do Amaral ${ }^{3}$
}

\begin{abstract}
RESUMO - Os biossólidos têm sido estudados como fonte de matéria orgânica na agricultura. Objetivou-se avaliar o desenvolvimento de mudas de Acacia mangium e Acacia auriculiformis em diferentes substratos: a) horizonte $\mathrm{Bw}$ com areia lavada $(1: 1, \mathrm{v}: \mathrm{v})$ e adubação mineral de 160,640 e $160 \mathrm{~g} \mathrm{~m}^{-3} \mathrm{de} \mathrm{N}, \mathrm{P}_{2} \mathrm{O}_{5}$ e $\mathrm{K}_{2} \mathrm{O}$, respectivamente (HB); b) horizonte $\mathrm{Bw}$ com areia lavada e esterco bovino (1:1:1, v:v) (HBE); c) horizonte Bw com areia lavada e lodo de esgoto (1:1:1, v:v) (HBL); e d) 100\% de lodo de esgoto (LE). Aplicou-se 1 $\mathrm{kg}$ de $\mathrm{CaCO}_{3}$ p.a. por $\mathrm{m}^{3}$ de substrato. Foram utilizadas sementes inoculadas com rizóbio e não-inoculadas, determinando-se, aos 90 dias após a semeadura, a altura das plantas, o diâmetro do colo e o peso da matéria seca da raiz e da parte aérea, na qual se determinaram N, P, K, Ca e Mg. O delineamento estatístico foi inteiramente casualizado, no esquema fatorial 2 x 4 (com ou sem inoculação x 4 substratos). No LE com inoculação, obtevese melhor crescimento das mudas. O HBE produziu efeito superior no desenvolvimento das mudas em relação àquele com a mesma proporção de material orgânico na forma de lodo (HBL). Na maioria dos parâmetros avaliados não houve diferença devido à inoculação dos substratos HBE, HBL e HB, provavelmente devido à existência de bactérias nativas nesses substratos. As mudas desenvolvidas no substrato LE foram as que acumularam mais $\mathrm{N}$ e $\mathrm{Ca}$, principalmente quando inoculadas. Houve tendência de maior acúmulo de $\mathrm{P}, \mathrm{K}$ e $\mathrm{Mg}$ na parte aérea das mudas desenvolvidas no substrato HBE.
\end{abstract}

Palavras-chave: esgoto doméstico, produção de mudas, leguminosa e rizóbio.

\section{EFFECT OF DIFFERENT SUBSTRATES ON THE DEVELOPMENT OF Acacia sp. SEEDLINGS}

\begin{abstract}
Sewage sludge has been studied as source of organic matter on seedling production. Thus, this study aimed to evaluate the development of Acacia mangium and Acacia auriculiformis seedlings in the following substrates: a) oxic horizon + sand $(1: 1, v: v)+160,640$ e $160 \mathrm{~g} \mathrm{~m}^{-3}$ of $\mathrm{N}, \mathrm{P}_{2} \mathrm{O}_{5}$ and $\mathrm{K}_{2} \mathrm{O}$ respectively $(H B) ; b)$ oxic horizon + sand + cattle manure $(1: 1: 1, v: v)(H B E) ; c)$ oxic horizon + sand + sewage sludge $(1: 1: 1, v: v)(H B L)$ and; d) $100 \%$ sewage sludge (LE). The substrates were limed with $1 \mathrm{~kg} \mathrm{~m}^{-3} \mathrm{CaCO}_{3}$ p.a.. Seeds, inoculated and non-inoculated with Rhizobium, were used, and 90 days after planting, measures of collar diameter, plant height, and root and shoot dry matter (with determination of $N, P, K, C a$ and $M g$ ) were taken. The experimental design was completely randomized blocks, in a factorial $2 \times 4$ arrangement (with or without inoculation $x 4$ substrates). Better performance in seedling growth was obtained in the LE
\end{abstract}

\footnotetext{
${ }^{1}$ Recebido em 05.10.2004 e aceito para publicação em 10.11.2005.

${ }^{2}$ Instituto Nacional de Colonização e Reforma Agrária - INCRA SR-26. AANE - 40, 1t 01/A, Alameda 1, 77010-050 Palmas-TO. E-mail: <alexson.cunha@pmj.incra.gov.br>.

${ }^{3}$ Departamento de Fitotecnia do Centro de Ciências Agrárias da Universidade Federal do Espírito Santo - CCAUFES, Caixa Postal 16, 29500-000 Alegre-ES. E-mail: <glaucioml@yahoo.com.br>.

${ }^{4}$ Departamento de Biologia Animal/Entomologia da Universidade Federal de Viçosa, 36570-000 Viçosa-MG, Brasil.

${ }^{5}$ Eng $^{\mathrm{a}}$. Agr ${ }^{\mathrm{a}}$. e estudante do Curso de Veterinária do CCAUFES, Caixa Postal 16, 29500-000 Alegre-ES.
} 
substrate with inoculated seeds. Comparatively, the HBE substrate was superior to the HBL substrate. There were no significant differences for most evaluated parameters in the $H B E, H B L$ and $H B$ substrates due to inoculation, probably because the existence of native bacteria in these substrates. The seedlings developing in the LE substrate accumulated more $N$ and $C a$, mainly when inoculated. There was a tendency of larger $P, K$ and $M g$ accumulation in shoots of seedlings developing in the HBE substrate.

Keywords: Domestic wastewater, seedling production, legume and Rhizobium.

\section{INTRODUÇÃO}

O substrato para a produção de mudas tem por finalidade garantir o desenvolvimento de uma planta com qualidade, em curto período de tempo, e baixo custo. A qualidade física do substrato é importante, por ser utilizado num estádio de desenvolvimento em que a planta é muito suscetível ao ataque por microrganismos e pouco tolerante ao déficit hídrico. Assim, o substrato deve reunir características físicas e químicas que promovam, respectivamente, a retenção de umidade e disponibilidade de nutrientes, de modo que atendam às necessidades da planta.

Na composição do substrato para o crescimento de plântulas, a fonte orgânica é responsável pela retenção de umidade e fornecimento de parte dos nutrientes. Tradicionalmente, o esterco bovino é utilizado como fonte orgânica na composição de substratos para viveiros de mudas de café, de plantas hortícolas e de plantas arbóreas (FONSECA, 1988; SANTOS et al., 1994b; ANDRADE NETO et al., 1999). No entanto, a disponibilidade do esterco bovino de qualidade depende da região e também do manejo das pastagens. O uso de herbicidas em pastagens, na maioria dos casos, inviabiliza o uso do esterco bovino na agricultura. No entanto, há disponível no mercado substratos para produção de mudas, mas que, dependendo da finalidade do uso, pode tornar-se inviável economicamente, como no caso de implantação de reflorestamento para recuperação de áreas degradadas.

No processo de produção de mudas de espécies florestais, o uso de lodo de esgoto é uma alternativa viável como fonte de matéria orgânica e de nutrientes para as plantas (MORAIS et al., 1996; MAIA, 1999; TELES et al., 1999). O emprego do lodo de esgoto como fonte de nutrientes para culturas agrícolas ainda apresenta alguma resistência, pelo receio da contaminação por patógenos (ANDREOLI et al., 1999). Em decorrência disso, o aproveitamento do lodo de esgoto para a produção

R. Árvore, Viçosa-MG, v.30, n.2, p.207-214, 2006 de espécies florestais tem sido estudado, principalmente em espécies indicadas para a recuperação de áreas degradadas (LOGAN et al., 1994; TELES et al., 1999).

As plantas leguminosas, em razão da grande diversidade de espécies, versatilidade de usos potenciais e de seu papel na dinâmica dos ecossistemas, especialmente quanto ao suprimento e ciclagem de nitrogênio $(\mathrm{N})$, têm sido indicadas para a recuperação de áreas degradadas (FRANCO et al., 1994; SANTOS et al., 1994a; KONDO e RESENDE, 2001). As leguminosas das espécies Acacia mangium e Acacia auriculiformis são originárias do nordeste da Austrália e Nova Guiné. As condições climáticas tropicais são quentes e úmidas ou subúmidas, com precipitação média anual de 1.000 a $2.000 \mathrm{~mm}$, podendo ser inferior para a espécie $A$. mangium. Os indivíduos dessas espécies atingem alturas entre 25 e $30 \mathrm{~m}$ e se desenvolvem bem em solos oxídicos argilosos, de baixa fertilidade e ácidos, podendo a $A$. mangium tolerar inclusive solos com impedimento de drenagem sazonal (TURNBULL, 1984).

O objetivo deste trabalho foi avaliar o desenvolvimento de mudas de $A$. mangium e $A$. auriculiformis, produzidas com substratos contendo lodo de esgoto e esterco de bovinos como fontes de matéria orgânica e de nutrientes, sob o efeito de inoculantes diazotróficos.

\section{MATERIAL E MÉTODOS}

\subsection{Material}

As fontes de materiais orgânicos utilizados para a composição dos substratos foram lodo de esgoto e esterco bovino. O lodo de esgoto foi proveniente da estação de tratamento de esgoto da cidade de Jerônimo Monteiro, ES, operada pelo Serviço Autônomo de Água e Esgoto (SAAE). O lodo de esgoto tratado foi de característica essencialmente doméstica. O esterco bovino, proveniente do estábulo do setor de Zootecnia, 
pertencente ao Centro de Ciências Agrárias da Universidade Federal do Espírito Santo (CCA/UFES).

O experimento foi conduzido em casa de vegetação do CCA/UFES, em Alegre, ES. Os substratos utilizados foram os seguintes: a) horizonte B latossólico com areia lavada (1:1, v:v) e adubação mineral na proporção de 160,640 e $160 \mathrm{~g}$ de $\mathrm{N}, \mathrm{P}_{2} \mathrm{O}_{5}$ e $\mathrm{K}_{2} \mathrm{O}$, respectivamente, por $\mathrm{m}^{3}$ de substrato, denominado $\mathrm{HB}$; b) horizonte $\mathrm{B}$ latossólico com areia lavada e esterco de bovino secado ao ar e peneirado em malha de $2 \mathrm{~mm}(1: 1: 1$, v:v), denominado HBE; c) horizonte B latossólico com areia lavada e lodo de esgoto (1:1:1, v:v), denominado HBL; e d) $100 \%$ de lodo de esgoto, denominado LE. Todos os substratos receberam calagem na dose de $1 \mathrm{~kg} \mathrm{~m}^{-3}$, utilizando-se $\mathrm{CaCO}_{3}$ p.a. Posteriormente, realizou-se a caracterização química dos substratos (Quadro 1), de acordo com a Embrapa (1997).

As sementes das espécies A. Mangium e $A$. auriculiformes foram coletadas em árvores do Horto Municipal da cidade de Alegre, sul do Estado do Espírito Santo.

\subsection{Método}

As sementes foram beneficiadas manualmente e submetidas, posteriormente, ao tratamento de escarificação com $\mathrm{H}_{2} \mathrm{SO}_{4}$, conforme De-Polli e Franco (1985). Previamente à semeadura, um conjunto de sementes foi inoculado com bactérias do gênero Rhizobium, de modo que se pudessem comparar substratos inoculados e não-inoculados. Os inoculantes, específicos para as espécies em estudo, foram fornecidos pela Embrapa/CNPAB.

A semeadura foi feita em 12 de fevereiro de 1998, nos substratos contidos em recipientes de bandejas de isopor tipo plantágil, com 72 células/bandeja. Em cada bandeja foram distribuídas três sementes de cada espécie por célula, perfazendo um total de 36 células por espécie. As células tinham 5 x $5 \mathrm{~cm}$ de área x 12 $\mathrm{cm}$ de altura no formato cônico. Foram distribuídas três sementes por recipiente. Decorridos 15 dias após a germinação, o desbaste foi efetuado, selecionandose a planta mais vigorosa.

Aos 90 dias após o semeio, avaliaram-se a altura e diâmetro do colo das mudas. As plantas foram destacadas da bandeja com os respectivos substratos e procedida, em seguida, à lavagem cuidadosa do sistema radicular. As plantas foram cortadas à altura do colo para separar parte aérea e raiz. As raízes e partes aéreas foram secadas a $60{ }^{\circ} \mathrm{C}$ até peso constante para a determinação da matéria seca, sendo a parte aérea triturada em moinho tipo Wiley, para posterior análise química.

As amostras de material vegetal da parte aérea foram submetidas à digestão sulfúrica para determinação do $\mathrm{N}$ total pelo método Kjeldhal (TEDESCO et al., 1985). Procedeu-se também à digestão nítrico-perclórica nas amostras para determinação de $\mathrm{Ca}$ e $\mathrm{Mg}$ por espectrofotometria de absorção atômica, de $\mathrm{P}$ por colorimetria e de K por fotometria de chama (TEDESCO et al., 1985). Os acúmulos dos macronutrientes $\mathrm{N}, \mathrm{P}$, $\mathrm{K}, \mathrm{Ca}$ e Mg na parte aérea das plantas foram obtidos, multiplicando-se o teor do nutriente pela massa seca correspondente.

A amostragem foi realizada no centro de cada bandeja de isopor, eliminando-se a bordadura. O número de repetições foi de 16 por bandeja, totalizando, assim, 256 plantas úteis. Para cada espécie, os dados foram analisados considerando-se o delineamento inteiramente casualizado, no esquema fatorial 2 × 4 (com inoculação e sem inoculação x 4 substratos). Procedeu-se à comparação de médias das variáveis, por meio do teste de Tukey a $5 \%$ de probabilidade.

Quadro 1 - Características químicas dos diferentes substratos avaliados Table 1 -Chemical characteristics of the different substrates

\begin{tabular}{|c|c|c|c|c|c|c|c|c|c|c|c|}
\hline \multirow[t]{2}{*}{ Substrato } & \multirow[t]{2}{*}{$\mathrm{pH}$} & $\mathrm{Ca}$ & $\mathrm{Mg}$ & $\mathrm{K}$ & $\mathrm{Na}$ & Al & $\mathrm{P}$ & $\mathrm{Cu}$ & $\mathrm{Zn}$ & $\mathrm{Fe}$ & $\mathrm{Mn}$ \\
\hline & & \multicolumn{5}{|c|}{$\mathrm{cmol}_{\mathrm{c}} \mathrm{dm}^{-3}$} & \multicolumn{5}{|c|}{$\mathrm{mg} \mathrm{dm}^{-3}$} \\
\hline $\mathrm{HB}$ & 5,1 & 0,9 & 0,4 & 0,04 & 0,03 & 0,0 & 24 & 1,0 & 3,1 & 56 & 23,2 \\
\hline HBE & 7,0 & 4,8 & 2,2 & 0,25 & 0,10 & 0,0 & 122 & 1,9 & 18,8 & 64 & 61,7 \\
\hline HBL & 5,8 & 2,6 & 0,8 & 0,06 & 0,07 & 0,0 & 108 & 5,2 & 13,6 & 283 & 24,8 \\
\hline LE & 5,1 & 5,0 & 0,9 & 0,10 & 0,11 & 0,1 & 143 & 11,9 & 49,2 & 478 & 29,6 \\
\hline
\end{tabular}

MO - Matéria orgânica; K, Na, P, Cu, Zn, Fe, Mn - extrator Melich 1; HB - horizonte B latossólico com areia lavada (1:1, v:v); HBE - horizonte B latossólico com areia lavada e esterco de bovino (1:1:1, v:v); HBL - horizonte B latossólico com areia lavada e lodo de esgoto (1:1:1, v:v); e LE - 100\% lodo de esgoto. 


\section{RESULTADOS E DISCUSSÃO}

\subsection{Avaliação de parâmetros morfológicos}

Em geral, em A. mangium o substrato constituído de lodo de esgoto puro (LE) apresentou resultados superiores apenas quando inoculado. Resultado semelhante também foi verificado em $A$. auriculiformis. Comparando-se os substratos, quando não-inoculados, o HBE proporcionou maior desenvolvimento na altura e diâmetro das mudas.

O efeito dos substratos e da inoculação sobre o desenvolvimento da A. mangium e da A. auriculiformis pode ser verificado no Quadro 2. O efeito favorável da inoculação em todos os parâmetros avaliados foi significativo apenas no substrato LE. No substrato HBL, a inoculação proporcionou efeito significativo apenas na altura da espécie $A$. mangium, sendo, no entanto, a altura da parte aérea um parâmetro muito utilizado e considerado um dos mais antigos e importantes para estimar o padrão de qualidade de mudas (GOMES et al., 1978; PARVIAINEN, 1981), e continua apresentando grande contribuição, como demonstrado por Gomes et al. (2002), em experimento sobre parâmetros morfológicos na avaliação de mudas de Eucaliptus grandis.
Entre os substratos avaliados, o LE inoculado promoveu maior crescimento em altura e maior produção de matéria seca da parte aérea e raiz na A. mangium, sendo, contudo, a medida de diâmetro sem diferença significativa com relação à do HBE inoculado. Isso indica certa semelhança entre os resultados de LE e HBE, pois, assim como a altura, o diâmetro do colo é também um dos mais importantes parâmetros morfológicos para estimar o crescimento de mudas (CARNEIRO, 1995). Muitas vezes se utiliza a combinação da altura e diâmetro, gerando um índice que fornece informações sobre quanto a muda está delgada (JOHNSON e CLINE, 1991).

Utilizando-se o substrato LE inoculado, houve um incremento de $227 \%$ na altura e de $150 \%$ no diâmetro do colo em relação aos valores encontrados no substrato HB inoculado. Esses resultados concordam com outros estudos que têm apontado benefícios do uso do lodo de esgoto na composição de substratos para mudas de diferentes espécies vegetais na fase de viveiro (MORAIS et al., 1996; TELES et al., 1999; TRIGUEIRO e GUERRINI, 2003; GUERRINI e TRIGUEIRO, 2004). Em A. auriculiformis, verificou-se maior altura nesse mesmo substrato LE inoculado, entretanto, no diâmetro do colo, matéria seca da parte aérea e da raiz, não houve diferença entre as mudas desenvolvidas nos substratos HBE e LE.

Quadro 2 - Médias de diâmetro do colo, altura, matéria seca da parte aérea (MSPA) e matéria seca da raiz (MSR) de mudas de $A$. mangium e A. auriculiformis, com inoculação $\left(\mathrm{C}_{\text {inoc }}\right)$ e sem inoculação $\left(\mathrm{S}_{\text {inoc }}\right)$

Table 2 - Average of collar diameter, shoot height, shoot dry matter (MSPA) and root dry matter (MSR) in A. mangium and A. auriculiformis seedlings, with $\left(C_{\text {inoc }}\right)$ and without inoculation $\left(S_{\text {inoc }}\right)$

\begin{tabular}{|c|c|c|c|c|c|c|c|c|}
\hline \multirow{3}{*}{ Substratos } & \multicolumn{2}{|c|}{ Altura } & \multicolumn{2}{|c|}{ Diâmetro } & \multicolumn{2}{|c|}{ MSPA } & \multicolumn{2}{|c|}{ MSR } \\
\hline & $\mathrm{C}_{\text {inoc }}$ & $\mathrm{S}_{\text {inoc }}$ & $\mathrm{C}_{\text {inoc }}$ & $\mathrm{S}_{\text {inoc }}$ & $\mathrm{C}_{\text {inoc }}$ & $\mathrm{S}_{\text {inoc }}$ & $\mathrm{C}_{\text {inoc }}$ & $\mathrm{S}_{\text {inoc }}$ \\
\hline & \multicolumn{4}{|c|}{$(\mathrm{cm})$} & \multicolumn{4}{|c|}{$\left(\mathrm{g} \cdot\right.$ planta $\left.^{-1}\right)$} \\
\hline & \multicolumn{8}{|c|}{ A. mangium } \\
\hline $\mathrm{HB}$ & $11,3 \mathrm{Ad}$ & $11,3 \mathrm{Ac}$ & $0,162 \mathrm{Ab}$ & $0,158 \mathrm{Ab}$ & $0,23 \mathrm{Ac}$ & $0,21 \mathrm{Ab}$ & $0,0580 \mathrm{Ac}$ & $0,0620 \mathrm{Ac}$ \\
\hline $\mathrm{HBE}$ & $20,3 \mathrm{Bb}$ & $22,6 \mathrm{Aa}$ & $0,234 \mathrm{Aa}$ & $0,253 \mathrm{Aa}$ & $0,63 \mathrm{Ab}$ & $0,57 \mathrm{Aa}$ & $0,2000 \mathrm{Ab}$ & $0,1580 \mathrm{Aa}$ \\
\hline HBL & $14,7 \mathrm{Ac}$ & $10,0 \mathrm{Bc}$ & $0,174 \mathrm{Ab}$ & $0,177 \mathrm{Ab}$ & $0,21 \mathrm{Ac}$ & $0,19 \mathrm{Ab}$ & $0,1730 \mathrm{Ab}$ & $0,1000 \mathrm{Ab}$ \\
\hline \multirow[t]{2}{*}{ LE } & $25,8 \mathrm{Aa}$ & $16,4 \mathrm{Bb}$ & $0,243 \mathrm{Aa}$ & $0,178 \mathrm{Bb}$ & $0,91 \mathrm{Aa}$ & $0,31 \mathrm{Bb}$ & $0,4730 \mathrm{Aa}$ & $0,1350 \mathrm{Ba}$ \\
\hline & \multicolumn{8}{|c|}{ A. auriculiformis } \\
\hline HB & $10,8 \mathrm{Ad}$ & $13,7 \mathrm{Ac}$ & $0,136 \mathrm{Bb}$ & $0,192 \mathrm{Aab}$ & $0,41 \mathrm{Ab}$ & $0,44 \mathrm{Ab}$ & $0,1080 \mathrm{Ab}$ & $0,1086 \mathrm{Ab}$ \\
\hline $\mathrm{HBE}$ & $21,6 \mathrm{Bb}$ & $25,1 \mathrm{Aa}$ & $0,201 \mathrm{Ba}$ & $0,236 \mathrm{Aa}$ & $0,84 \mathrm{Aa}$ & $0,88 \mathrm{Aa}$ & $0,1851 \mathrm{Aa}$ & $0,1901 \mathrm{Aa}$ \\
\hline $\mathrm{HBL}$ & $16,1 \mathrm{Ac}$ & $18,1 \mathrm{Ab}$ & $0,160 \mathrm{Ab}$ & $0,187 \mathrm{Ab}$ & $0,48 \mathrm{Ab}$ & $0,54 \mathrm{Ab}$ & $0,1869 \mathrm{Aa}$ & $0,1897 \mathrm{Aa}$ \\
\hline LE & 25,9 Аa & $22,0 \mathrm{Bab}$ & $0,226 \mathrm{Aa}$ & $0,202 \mathrm{Aab}$ & $0,86 \mathrm{Aa}$ & 0,90 Aa & $0,1437 \mathrm{Aab}$ & $0,1459 \mathrm{Aab}$ \\
\hline
\end{tabular}

As médias seguidas da mesma letra maiúscula na linha e da mesma letra minúscula na coluna não diferem entre si, estatisticamente, pelo teste de Tukey a $5 \%$ de probabilidade.

R. Árvore, Viçosa-MG, v.30, n.2, p.207-214, 2006 
$\mathrm{Na}$ ausência de inoculação, o substrato HBE condicionou maior crescimento em altura, diâmetro do colo e matéria seca da parte aérea na $A$. mangium e maior altura e diâmetro do colo na A. auriculiformis, embora, em relação ao LE sem inoculação, não se tenha verificado diferença na produção de matéria seca de parte aérea e da raiz. Nesses casos, é possível ter ocorrido uma população rizobiana nativa, conforme sugerido por Holanda et al. (1982a) ao estudar a fixação do nitrogênio atmosférico em plantas de sabiá (Mimosa caesalpiniifolia Benth). Outrossim, Chaves et al. (2003) não verificaram incrementos em altura e diâmetro de mudas de Sesbania virgata, cujas sementes foram inoculadas por rizóbio.

O substrato com lodo de esgoto (HBL), ainda que preparado na mesma proporção do substrato contendo esterco bovino (HBE), não condicionou o mesmo desenvolvimento às mudas das duas espécies de leguminosas (Quadro 2). Embora esse resultado evidencie a necessidade de uma dose de lodo maior que $1 / 3$ na composição do substrato, Morais et al. (1996), ao compararem esterco bovino, biossólido e acículas de pinus, comprovaram que o melhor crescimento em altura total e diâmetro para mudas de cedro (Cedrella fissilis Vell) foi obtido utilizando substrato com $70 \%$ de solo +30\% de biossólido, sem adubação. Em outro estudo, Trigueiro e Guerrini (2003), ao compararem substratos contendo biossólido + casca de arroz carbonizada e

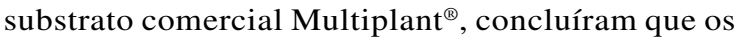
melhores resultados foram obtidos com substratos com proporção de 40 a $50 \%$ de biossólido em mistura com casca de arroz carbonizada.

Verificou-se que nas mudas com inoculação os maiores diâmetros foram obtidos quando elas se desenvolveram nos substratos LE e HBE (Quadro 2), sendo que neste último, nas espécies $A$. mangium e A. auriculiformis, os valores de diâmetro do colo foram, respectivamente, 145 e $148 \%$ superiores ao substrato HB. As médias de diâmetro do colo não se diferiram nos substratos HB e HBL. Na ausência de inoculação, o substrato HBE condicionou maior diâmetro do colo às mudas.

Segundo Cordell e Filler (1984), citados por Rosa Junior et al. (1998), a matéria orgânica é considerada um componente fundamental em substratos. A ausência de fonte orgânica para a composição do substrato HB condicionou menor desenvolvimento da parte aérea e raiz das mudas das duas espécies avaliadas. Tal observação indica que a ausência de fonte orgânica não proporcionou condições físicas e químicas adequadas para o desenvolvimento das mudas, apesar de o substrato HB conter nutrientes fornecidos pela adubação e calagem.

De acordo com os resultados observados a respeito do desenvolvimento das mudas de A. mangium e A. auriculiformis, o substrato de HBE, com a proporção de $1 / 3$ de esterco, produziu efeito equivalente à aplicação do lodo de esgoto $100 \%$ como substrato. No entanto, a utilização do lodo de esgoto na proporção de $1 / 3$ na composição do substrato HBL foi insuficiente para exercer efeito positivo no diâmetro e matéria seca nas duas espécies.

\subsection{Acúmulo de macronutrientes}

O conteúdo de macronutrientes da parte aérea de mudas de A. mangium e A. auriculiformes pode ser observado no Quadro 3. Conforme Holanda et al. (1982b), o nitrogênio total das plantas é um dos parâmetros que melhor expressa a eficiência na fixação do $\mathrm{N}$ atmosférico. De modo geral, houve tendência de as mudas desenvolvidas no substrato LE acumularem mais $\mathrm{N}$, mesmo na ausência de inoculação. A inoculação dos substratos proporcionou maiores conteúdos de $\mathrm{N}$ nas plantas desenvolvidas nos substratos LE, seguido do HBE e HBL. Os valores encontrados para $\mathrm{N}$ nas mudas de $A$. mangium e $A$. auriculiformis inoculadas produzidas no substrato LE foram, respectivamente, 405 e $413 \%$ maiores que nas mudas obtidas no substrato HB.

As mudas no substrato HB acumularam menos $\mathrm{N}$, provavelmente em resposta à ausência de matéria orgânica mineralizável e à baixa eficiência das bactérias na fixação do $\mathrm{N}$ atmosférico em ambiente de baixa fertilidade. A menor disponibilidade de $\mathrm{P}$ foi verificada no substrato HB (Quadro 1), assim como o menor conteúdo na parte aérea (Quadro 3). Segundo Holanda et al. (1982b) e Rocha e Holanda (1983), ao estudarem a fixação de $\mathrm{N}_{2}$, respectivamente em Clitoria ternatea e Stylosanthes hamata, concluíram que o P no solo foi o nutriente que mais limitou a fixação biológica. Apenas o substrato HB não respondeu à inoculação, sendo esse e o HBL, para a espécie $A$. auriculiformis, com maiores acúmulos de $\mathrm{N}$ quando não inoculados.

R. Árvore, Viçosa-MG, v.30, n.2, p.207-214, 2006 
Quadro 3 - Médias dos teores de macronutrientes na parte aérea de mudas de A. mangium e A. auriculiformis, com inoculação $\left(\mathrm{C}_{\text {inoc }}\right)$ e sem inoculação $\left(\mathrm{S}_{\text {inoc }}\right)$

Table 3 - Average of macronutrient contents in shoots of A.mangium and A. auriculiformis seedlings, with $\left(C_{\text {inoc }}\right)$ and without inoculation $\left(S_{\text {inoc }}\right)$

\begin{tabular}{|c|c|c|c|c|c|c|c|c|c|c|}
\hline \multirow[t]{2}{*}{ Substrato } & \multicolumn{2}{|c|}{$\mathrm{N}$} & \multicolumn{2}{|c|}{$\mathrm{P}$} & \multicolumn{2}{|c|}{$\mathrm{K}$} & \multicolumn{2}{|c|}{$\mathrm{Ca}$} & \multicolumn{2}{|c|}{$\mathrm{Mg}$} \\
\hline & $\mathrm{C}_{\text {inoc }}$ & $\mathrm{S}_{\mathrm{inoc}}$ & $\mathrm{C}_{\text {inoc }}$ & $\mathrm{S}_{\mathrm{inoc}}$ & $\mathrm{C}_{\text {inoc }}$ & $\mathrm{S}_{\text {inoc }}$ & $\mathrm{C}_{\text {inoc }}$ & $\mathrm{S}_{\mathrm{inoc}}$ & $\mathrm{C}_{\text {inoc }}$ & $\mathrm{S}_{\text {inoc }}$ \\
\hline & \multicolumn{10}{|c|}{ mg.planta ${ }^{-1}$} \\
\hline & \multicolumn{10}{|c|}{ A. mangium } \\
\hline $\mathrm{HB}$ & $5,33 \mathrm{Ac}$ & 4,92 Abc & $0,66 \mathrm{Ad}$ & $0,72 \mathrm{Ac}$ & $2,40 \mathrm{Ad}$ & $2,98 \mathrm{Ad}$ & $2,94 \mathrm{Ac}$ & $3,49 \mathrm{Ab}$ & $1,04 \mathrm{Ac}$ & $0,68 \mathrm{Bb}$ \\
\hline HBE & $10,32 \mathrm{Ab}$ & 7,29 $\mathrm{Bab}$ & $4,45 \mathrm{Aa}$ & 4,92 Аа & $11,95 \mathrm{Aa}$ & $9,17 \mathrm{Aa}$ & $8,02 \mathrm{Ab}$ & $7,04 \mathrm{Aa}$ & $3,34 \mathrm{Aa}$ & $3,10 \mathrm{Aa}$ \\
\hline HBL & $8,68 \mathrm{Ab}$ & $5,40 \mathrm{Bbc}$ & $2,30 \mathrm{Ac}$ & $1,75 \mathrm{Bb}$ & $5,29 \mathrm{Ac}$ & $4,17 \mathrm{Ac}$ & $4,09 \mathrm{Ac}$ & $2,90 \mathrm{Ab}$ & $0,86 \mathrm{Ac}$ & $0,50 \mathrm{Bb}$ \\
\hline \multirow[t]{2}{*}{ LE } & $21,60 \mathrm{Aa}$ & $9,17 \mathrm{Ba}$ & $3,64 \mathrm{Ab}$ & $1,33 \mathrm{Bbc}$ & $7,03 \mathrm{Ab}$ & $6,41 \mathrm{Ab}$ & $13,07 \mathrm{Aa}$ & $4,11 \mathrm{Bb}$ & $2,54 \mathrm{Ab}$ & $0,85 \mathrm{Bb}$ \\
\hline & \multicolumn{10}{|c|}{ A. auriculiformis } \\
\hline $\mathrm{HB}$ & $6,99 \mathrm{Bc}$ & $11,91 \mathrm{Ab}$ & $1,03 \mathrm{Ac}$ & $1,01 \mathrm{Ac}$ & $2,38 \mathrm{Ac}$ & $3,40 \mathrm{Ac}$ & $6,01 \mathrm{Ab}$ & $4,84 \mathrm{Ab}$ & $1,38 \mathrm{Ab}$ & $1,78 \mathrm{Ab}$ \\
\hline HBE & $14,22 \mathrm{Ab}$ & $11,66 \mathrm{Ab}$ & $4,30 \mathrm{Ba}$ & $5,13 \mathrm{Aa}$ & $9,11 \mathrm{Ba}$ & $16,94 \mathrm{Aa}$ & $8,66 \mathrm{Ab}$ & $7,72 \mathrm{Ab}$ & $2,86 \mathrm{Aa}$ & $3,42 \mathrm{Aa}$ \\
\hline HBL & $11,52 \mathrm{Bbc}$ & $16,09 \mathrm{Aab}$ & $2,14 \mathrm{Ab}$ & $2,10 \mathrm{Ab}$ & $6,08 \mathrm{Ab}$ & $6,40 \mathrm{Ab}$ & $7,13 \mathrm{Ab}$ & $5,18 \mathrm{Ab}$ & $1,11 \mathrm{Ab}$ & $1,60 \mathrm{Ab}$ \\
\hline LE & $28,89 \mathrm{Aa}$ & $20,49 \mathrm{Ba}$ & $2,88 \mathrm{Ab}$ & $2,05 \mathrm{Bb}$ & $6,20 \mathrm{Ab}$ & $5,25 \mathrm{Abc}$ & $12,08 \mathrm{Ba}$ & $14,86 \mathrm{Aa}$ & $2,83 \mathrm{Aa}$ & $2,50 \mathrm{Aa}$ \\
\hline
\end{tabular}

As médias seguidas da mesma letra maiúscula na linha e da mesma letra minúscula na coluna não diferem entre si, estatisticamente, pelo teste de Tukey a $5 \%$ de probabilidade.

Teores razoáveis de nutrientes como $\mathrm{Ca}, \mathrm{Mg}, \mathrm{S}$ e com destaque $\mathrm{N}$ e $\mathrm{P}$ são encontrados em biossólidos de origens residencial e industrial, conforme constatado por Guerrini e Trigueiro (2004), ao estudá-los na produção de mudas de Eucaliptus grandis. No presente estudo, conteúdos mais elevados dos macronutrientes $\mathrm{P}$ e $\mathrm{K}$ foram encontrados no substrato HBE, seguido do LE. Os menores conteúdos de $\mathrm{K}$ nas mudas desenvolvidas no substrato LE, em relação ao HBE, deveram-se ao fato de a menor disponibilidade desse nutriente no lodo. Segundo Oliveira et al. (1995), o lodo de esgoto possui baixa concentração de $\mathrm{K}$, em razão da sua alta solubilidade em água, sendo necessária a complementação do lodo com esse nutriente.

No que se refere ao $\mathrm{Mg}$, o substrato $\mathrm{HBE}$ foi superior ao LE apenas na espécie $A$. mangium. Quanto ao Ca, o substrato LE sobressaiu, exceto na $A$. mangium sem inoculação. Apesar de os substratos HBE e LE apresentarem teores semelhantes de cálcio (Quadro 1), a parte aérea das plantas desenvolvidas no primeiro exibiram menor acúmulo de cálcio (Quadro 3), provavelmente devido ao efeito antagônico entre Ca e K. Esse efeito foi verificado por Trigueiro e Guerrini (2003), porém com altos teores de Ca, reduzindo a absorção de K em mudas de Eucaliptus grandis.

Os maiores conteúdos de $\mathrm{P}, \mathrm{K}, \mathrm{Ca}$ e $\mathrm{Mg}$ na parte aérea de plantas de $A$. mangium e $A$. auriculiformis nos substratos HBE e LE (Quadro 3) refletiram as disponibilidades desses nutrientes, conforme observado na caracterização química dos substratos (Quadro 1).

\section{CONCLUSÕES}

O substrato composto de lodo de esgoto $100 \%$ (LE) com sementes inoculadas proporcionou maior desenvolvimento às mudas de $A$. mangium e $A$. auriculiformis.

A melhor "performance" das mudas, comparandose diferentes substratos com a mesma proporção de material orgânico, foi observada quando se utilizou esterco bovino.

Mudas produzidas nos substratos HBE, HBL e HB não responderam à inoculação na maioria dos parâmetros avaliados, provavelmente devido à existência de bactérias nativas nesse substrato.

As mudas desenvolvidas no substrato LE foram as que acumularam mais $\mathrm{Ne} \mathrm{Ca}$, principalmente quando inoculadas. Houve tendência de maior acúmulo de $\mathrm{P}$, $\mathrm{K} \mathrm{e} \mathrm{Mg} \mathrm{em} \mathrm{mudas} \mathrm{crescidas} \mathrm{no} \mathrm{substrato} \mathrm{com} \mathrm{esterco}$ bovino.

\section{REFERÊNCIAS BIBLIOGRÁFICAS}

ANDRADE NETO, A.; MENDES, A.N.G.; GUIMARÃES, P.T.G. Avaliação de substratos alternativos e tipo de adubação para a produção de mudas de cafeeiro em tubetes. Ciência e Agrotecnologia, v. 23, n. 2, p.270-280, 1999. 
ANDREOLI, C.V. et al. Aceitabilidade pública da utilização do lodo de esgoto na agricultura da região metropolitana de Curitiba. Sanare, v. 12, n. 12, p.43-52, 1999.

CARneiro, J.G.A. Produção e controle de qualidade de mudas florestais. Curitiba: Universidade Federal do Paraná: Campos dos Goytacazes: Universidade Estadual do Norte Fluminense, 1995. 451p.

CHAVES, L. L. B. et al. Efeitos da inoculação com rizóbio e da adubação nitrogenada na produção de mudas de Sesbânia em substrato constituído de resíduos agroindustriais. Revista

Árvore. v. 27, n. 4, p.443-449, 2003.

DE-POLLI, H.; FRANCO, A.A. Inoculação de sementes de leguminosas. Seropédica: EMBRAPA/UAPNPBS, 1985. 31p. (EMBRAPA/ UAPNPBS. Circular Técnica, 1).

Empresa Brasileira de Pesquisa Agropecuária EMBRAPA. Centro Nacional de Pesquisa de Solos. Manual de métodos de análise de solo. 2. ed. Rio de Janeiro: 1997. 212p.

FONSECA, E.P. Efeito de diferentes substratos na produção de mudas de Eucalyptus grandis W. Hill ex Maiden em “Win-strip". 1988. 81f. Dissertação (Mestrado em Ciência Florestal) - Universidade Federal de Viçosa, Viçosa, 1988.

FRANCO, A.A. et al. Revegetação de áreas de mineração de bauxita em Porto Trombetas-PA com leguminosas arbóreas noduladas e micorrizadas. In: SIMPÓSIO SUL-AMERICANO, 1; SIMPÓSIO NACIONAL DE RECUPERAÇÃO DE ÁREAS DEGRADADAS, 2., 1994, Foz do Iguaçu. Anais...Curitiba: Universiade Federal do Paraná, 1994. p.145-153.

GOMES, J. M. et al. Influência do tratamento prévio do solo com brometo de metila no crescimento de mudas de Pinus caribaea var. hondurensis em viveiro. Brasil Florestal, v. 9, n. 35 , p. $18-23,1978$.

GOMES, J.M. et al. Parâmetros morfológicos na avaliação da qualidade de mudas de Eucaliptus grandis. Revista Árvore, v. 26, n. 6, p. 655664, 2002.

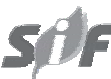

GUERRINI, I.A.; TRIGUEIRO, R.M. Atributos físicos e químicos de substratos compostos por biossólidos e casca de arroz carbonizada.

Revista Brasileira de Ciência do Solo, v. 28, n. 6, p. 1069-1076, 2004.

HOLANDA, F.J.M.; VASCONCELOS, I.; OLIVEIRA, M.E.S. Fatores nutricionais limitantes da fixação de nitrogênio atmosférico em cunhã (Clitoria ternatea L.). Revista Ciência Agronômica, v. 13, n. 1/2, p. 29-33, 1982 b.

HOLANDA, F.J.M.; VASCONCELOS, I.; OLIVEIRA, M.E.S. Fatores nutricionais limitantes da fixação de nitrogênio atmosférico em sabiá (Mimosa caesalpineaefolia Benth.). Revista Ciência Agronômica, v. 13, n.1/2, p.29-33. 1982a.

JOHNSON, J. D.; CLINE, P. M. Seedling quality of southern pines. In: DUREYA, M. L., DOUGHERTY, P. M. (Ed.). Forest regeneration manual. Dordrecht: Kluwer Academic Publishers, 1991. p.143-162.

KONDO, M.K.; RESENDE, A.V. Recuperação de pastagens degradadas. Informe

Agropecuário, v.22, n. 210, p.36-45, 2001.

LOGAN, T.J. et al. Recuperação de áreas ácidas mineradas de carvão através da utilização de lodo de esgoto com avançada estabilização alcalina, Nviro soil. In. SIMPÓSIO SUL-AMERICANO, 1., e SIMPÓSIO NACIONAL DE RECUPERAÇÃO DE ÁREAS DEGRADADAS, 2., 1994, Foz do Iguaçu. Anais... Curitiba: Universidade Federal do Paraná, 1994. p.313-323.

MAIA, C.M.B.F. Uso de casca de Pinus e lodo biológico como substrato para produção de mudas de Pinus taeda. Boletim de Pesquisa Florestal, v.3 9, p.81-92, 1999.

MORAIS, J.M.J. et al. Uso de lodo de esgoto da Corsan - Santa Maria (RS), comparado com outros substratos orgânicos. Sanare, v. 6, n. 6, p. 44-49, 1996.

OLIVEIRA, F.C. et al. Lodo de esgoto como fonte de macronutrientes para a cultura do sorgo granífero. Scientia Agrícola, v.52, n. 2, p.360-367, 1995.

R. Árvore, Viçosa-MG, v.30, n.2, p.207-214, 2006 
PARVIAINEN, J. V. Qualidade e avaliação de qualidade de mudas florestais. In: SEMINÁRIO DE SEMENTES E VIVEIROS FLORESTAIS, 1. ., 1981, Curitiba. Anais... Curitiba: Universidade Federal do Paraná, 1981. p. 59-90.

ROCHA, D. R.; HOLANDA, F.J.M. Efeitos da nutrição mineral e da inoculação na fixação simbiótica do nitrogênio atmosférico em Stylosanthes hamata (L.) Taub. cv. verano. Revista Ciência Agronômica, v. 14, n. 1/2, p. 97-105, 1983 .

ROSA JUNIOR, E.J. et al. Efeitos de diferentes substratos sobre o desenvolvimento de mudas de Eucaliptus grandis Hill, em tubetes. Revista de Ciências Agrárias, v. 1, n. 2, p. 18-22, 1998.

SANTOS, C.J.F. et al. Uso de leguminosas arbóreas no reflorestamento de encosta de risco geotécnico sobre comunidade de baixa renda. In. SIMPÓSIO SUL-AMERICANO, 1., SIMPÓSIO NACIONAL DE RECUPERAÇÃO DE ÁREAS DEGRADADAS, 2., 1994, Foz do Iguaçu.

Anais...Curitiba: Universidade Federal do Paraná, 1994a. v. 1, p. 361-369.
SANTOS, L.P.; CARVALHO, M.M.; CARVALHO, J.G. Efeitos de doses de nitrato de potássio e esterco de curral na composição do substrato para a formação de cafeeiro. Ciência e Prática, v. 18, n. 1, p. 42-48, 1994b.

TEDESCO, M.J.; VOLKWEISS, S.J.; BOHNEN, H. Análises de solo, plantas e outros materiais. Porto Alegre: Universidade Federal do Rio Grande do Sul, 1985. 50p. (Boletim Técnico, 5)

TRIGUEIRO, R.M.; GUERRINI, I.A. Uso de biossólidos como substratos para produção de mudas de eucalipto. Scientia Florestalis, n.164, p. 150-162, 2003.

TELES, C.R.; COSTA, A.N.; GONCALVES, R.F. Produção de lodo de esgoto em lagoas de estabilização e o seu uso no cultivo de espécies florestais na região sudoeste do Brasil. Sanare, v.12, n. 12, p. 53-60, 1999.

TURNBULL, J.W. Seis espécies de acácia para regiões do trópico úmido. Pesquisa

Agropecuária Brasileira, v.19, p. 69-73, 1984. 